\title{
A New Sign Distance-Based Ranking Method for Fuzzy Numbers
}

\author{
Kok Chin Chai ${ }^{1}$, Kai Meng Tay ${ }^{1}$, and Chee Peng Lim $^{2}$ \\ ${ }^{1}$ Universiti Malaysia Sarawak, Kota Samarahan, Sarawak, Malaysia \\ ${ }^{2}$ Centre for Intelligent Systems Research, Deakin University, Australia \\ kcchai@live.com, kmtay@feng.unimas.my
}

\begin{abstract}
In this paper, a new sign distance-based ranking method for fuzzy numbers is proposed. It is a synthesis of geometric centroid and sign distance. The use of centroid and sign distance in fuzzy ranking is not new. Most existing methods (e.g., distance-based method [9]) adopt the Euclidean distance from the origin to the centroid of a fuzzy number. In this paper, a fuzzy number is treated as a polygon, in which a new geometric centroid for the fuzzy number is proposed. Since a fuzzy number can be represented in different shapes with different spreads, a new dispersion coefficient pertaining to a fuzzy number is formulated. The dispersion coefficient is used to fine-tune the geometric centroid, and subsequently sign distance from the origin to the tuned geometric centroid is considered. As discussed in [5-9], an ideal fuzzy ranking method needs to satisfy seven reasonable fuzzy ordering properties. As a result, the capability of the proposed method in fulfilling these properties is analyzed and discussed. Positive experimental results are obtained.
\end{abstract}

Keywords: Geometric centroid, sign distance, fuzzy numbers, reasonable ordering properties, dispersion coefficient.

\section{Introduction}

Fuzzy ranking attempts to order a set of fuzzy numbers. The importance of fuzzy ranking in many application domains has been highlighted in the literature, e.g., decision-making [1], data analysis [2], risk assessment [3] and artificial intelligence [4]. Indeed, many fuzzy ranking methods have also been developed [1][5][6]. These methods can be categorized into three categories [5-6]; i.e., (1) transforming a set of fuzzy numbers into crisp numbers and subsequently ranking the crisp numbers; (2) mapping a set of fuzzy numbers to crisp numbers based on a pre-defined reference set(s) for comparison; (3) ranking through pairwise comparison of fuzzy numbers.

In this paper, our focus is on the first category, which is straightforward as compared with the other two categories. Nevertheless, the challenge is to develop a method that satisfies a set of reasonable ordering properties [5-9], as detailed in Section 2.2. Recently, a number of methods, e.g., deviation degree-based [8][10], distancebased [9][11], centroid-based [12], and area-based [13], have been proposed. However, many of these methods [8][10-13] do not have analysis pertaining to fulfillment 
of the reasonable ordering properties [5-9]. Besides that, fuzzy ranking is complicated when fuzzy numbers are presented as different shapes with different spreads, e.g., as illustrated in Fig. 1 [8].

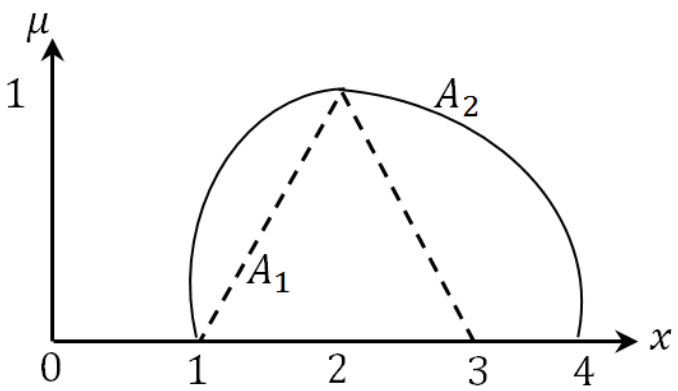

Fig. 1. Triangular (i.e., $A_{1}$ ) and generalized (i.e., $A_{2}$ ) fuzzy numbers [8]

In this paper, a new fuzzy ranking method that deals with fuzzy numbers (Definition 2), which is a synthesis of geometric centroid and sign distance, is proposed. The proposed method is capable of handling more complex fuzzy numbers i.e., generalized fuzzy numbers, as illustrated in Fig. 1. The method can be summarized into a few steps, as follows: (1) Fuzzy numbers are firstly converted into normalized fuzzy numbers (i.e., a set of fuzzy numbers available in space $R^{*} \in[-1,1]$ ). (2) A discretization technique [14], i.e., slicing the support of each normalized fuzzy number into $N$ slices vertically at even intervals, is employed. The intersections between the normalized fuzzy number and the $N$ vertical slices are called intersection points or discretized points. (3) Using the discretized points, geometric centroid of the normalized fuzzy number is then computed using Bourke's method [15]. To deal with more complex fuzzy numbers, as depicted in Fig. 1, a new dispersion coefficient of the normalized fuzzy numbers is proposed. The dispersion coefficient attempts to fine-tune the geometric centroid. (4) Finally, sign distance between the origin and the tuned geometric centroid is measured. In this paper, the results obtained from the sign distances are used to order a set of fuzzy numbers. The fulfilment of the seven reasonable ordering properties as stated in [5-9] is further examined. To evaluate the proposed method, benchmark examples from [8] are used.

The rest of the paper is organized as follows. In Section 2, some background studies are presented. In Section 3, the proposed ranking method is explained. In Section 4, an experimental study and the associated results are presented. Finally, concluding remarks are given in Section 5.

\section{Background}

\subsection{Definitions}

The following mathematical notations are widely used. $R$ denotes a set of real numbers, $\mu$ represents a fuzzy number, and $\mu(x)$ for its membership function, $\forall x \in R$. Definition 1, as follows, is considered. 
Definition 1 [16]: A fuzzy number, $A$ is a fuzzy set such that $\mu: R \rightarrow[0,1]$ which satisfies the following properties:

- $A$ is represented as $\left(a_{1}, a_{2}, a_{3}, a_{4}\right)$

- $\mu$ is upper semi-continuous

- $a_{1}, a_{2}, a_{3}$ and $a_{4}$ are real numbers, in such $a_{1} \leq a_{2} \leq a_{3} \leq a_{4} . \mu$ is strictly increasing for interval $\left[a_{1}, a_{2}\right]$ and strictly decreasing for interval $\left[a_{3}, a_{4}\right]$.

- $\mu(x)=1$, for $a_{2} \leq x \leq a_{3}$,

- $\mu(x)=0$ for $x$ not in the interval of $\left[a_{1}, a_{4}\right]$,

- Support of $A$, i.e., $\sup (A)=\{x \in R \mid \mu(x)>0\}$.

- $\mu$ is expressed as follows:

$$
\mu(x)=\left\{\begin{array}{c}
\mu^{L}(x), a_{1} \leq x \leq a_{2}, \\
1, a_{2} \leq x \leq a_{3} \\
\mu^{R}(x), a_{3} \leq x \leq a_{4} \\
0, \text { otherwise. }
\end{array}\right.
$$

where $\mu^{L}:\left[a_{1}, a_{2}\right] \rightarrow[0,1]$ and $\mu^{R}:\left[a_{3}, a_{4}\right] \rightarrow[0,1]$ are left and right membership functions of fuzzy number $u$. If $\mu^{L}(x)$ and $\mu^{R}(x)$ are linear functions and $a_{2}<a_{3}$, then $A$ is a trapezoidal fuzzy number. If $\mu^{L}(x)$ and $\mu^{R}(x)$ are linear functions, $a_{2}=a_{3}$, then $A$ is a special case of a trapezoidal fuzzy number i.e., a triangular fuzzy number.

Definition 2 [17]: Normalized fuzzy numbers (i.e., $A_{i}^{*}, i=1,2,3, \ldots, m$ ) are a set of fuzzy numbers in space $R^{*} \in[-1,1]$. Consider a set of fuzzy numbers $A_{i}$ where $i=1,2,3, \ldots, m$ in space $R \in(-\infty, \infty)$. $A_{i}^{*}$ is obtained with Eq. (2).

$$
A_{i}^{*}=\left(\frac{a_{i 1}}{k}, \frac{a_{i 2}}{k}, \frac{a_{i 3}}{k}, \frac{a_{i 4}}{k}\right)=\left(a_{i 1}^{*}, a_{i 2}^{*}, a_{i 3}^{*}, a_{i 4}^{*}\right)
$$

where $k=\max \left(\left|a_{i j}\right|, 1\right),\left|a_{i j}\right|$ is the absolute value of $a_{i j}, 1 \leq i \leq m$ and $1 \leq j \leq 4$. As an example, $A_{1}^{*}=\left(a_{11}^{*}, a_{12}^{*}, a_{13}^{*}, a_{14}^{*}\right)$ is illustrated in Fig.2, and centroids of $A_{1}$ is written as $c X_{A_{1}^{*}}, c Y_{A_{1}^{*}}$.

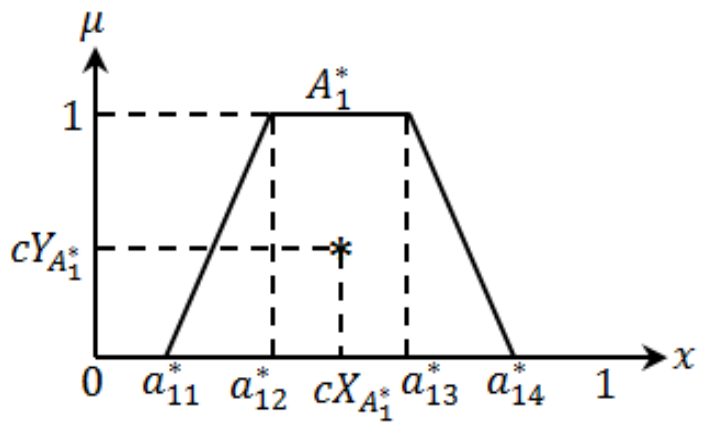

Fig. 2. The membership function of a normalized trapezoidal fuzzy number 


\subsection{Seven Reasonable Ordering Properties}

Consider three fuzzy numbers, i.e., $A_{1}, A_{2}$, and $A_{3}$ in space $R$. An ideal fuzzy ranking method should satisfy seven reasonable ordering properties, as follows. The first six reasonable ordering properties (i.e., P1-P6) are introduced in [5-6], while P7 is introduced in recent literatures [7-9].

P1: If $A_{1} \geqslant A_{2}$ and $A_{2} \geqslant A_{1}$, then $A_{1} \sim A_{2}$.

P2: If $A_{1} \geqslant A_{2}$ and $A_{2} \geqslant A_{3}$, then $A_{1} \geqslant A_{3}$.

P3: If $A_{1} \cap A_{2}=\emptyset$ and $A_{1}$ is on the right of $A_{2}$, then $A_{1} \geqslant A_{2}$.

P4: The order of $A_{1}$ and $A_{2}$ is not affected by other fuzzy numbers under comparison.

P5: If $A_{1} \geqslant A_{2}$, then $A_{1}+A_{3} \geqslant A_{2}+A_{3}$.

P6: If $A_{1} \geqslant A_{2}$, then $A_{1} A_{3} \geqslant A_{2} A_{3}$.

P7: If $A_{1} \geqslant A_{2} \geqslant A_{3}$ then $-A_{1} \preccurlyeq-A_{2} \preccurlyeq-A_{3}$.

This paper focuses on fulfillment of these seven reasonable ordering properties for trapezoidal fuzzy numbers. Note that the addition and multiplication operators in P5 and $\mathbf{P 6}$ are based on fuzzy arithmetic operators as follows:

Fuzzy Addition operator [11]:

$$
\begin{aligned}
A_{1} \oplus A_{2}=\left(a_{11}, a_{12}, a_{13}, a_{14}\right) \oplus\left(a_{21}, a_{22}, a_{23}, a_{24}\right) \\
=\left(a_{11}+a_{21}, a_{12}+a_{22}, a_{13}+a_{23}, a_{14}+a_{24}\right)
\end{aligned}
$$

Fuzzy Multiplication [11]:

$$
\begin{aligned}
A_{1} \otimes A_{2}=\left(a_{11}, a_{12}, a_{13}, a_{14}\right) \otimes\left(a_{21}, a_{22}, a_{23}, a_{24}\right) \\
=\left(a_{11} \times a_{21}, a_{12} \times a_{22}, a_{13} \times a_{23}, a_{14} \times a_{24}\right)
\end{aligned}
$$

Fuzzy Image [7]-[9]:

Fuzzy image of $A_{1}=\left(a_{11}, a_{12}, a_{13}, a_{14}\right)$ is $-A_{1}=\left(-a_{14},-a_{13},-a_{12},-a_{11}\right)$.

\section{The Proposed Methodology}

Consider $m$ fuzzy numbers, $A_{i}$, i.e., $A_{1}, A_{2}, \ldots, A_{m}$, in space $R \in[-\infty, \infty]$, which have to be ranked. The proposed method is summarized into five steps as follows:

Step 1: Transform each fuzzy number $A_{i}=\left(a_{i 1}, a_{i 2}, a_{i 3}, a_{i 4}\right)$ into a normalized fuzzy number $A_{i}^{*}=\left(a_{i 1}^{*}, a_{2 i}^{*}, a_{i 3}^{*}, a_{i 4}^{*}\right)$ using Eq. (2).

Step 2: Discretize the support of $A_{i}^{*}$ into $N$ points, i.e., $x_{i j}^{*}, j=1,2,3 \ldots, N$, and obtain $\mu_{A_{i}^{*}}\left(x_{i j}^{*}\right)$, where $x_{i j}^{*} \in R^{*}$. The discretized points are expressed in a sequence of $x_{i 1}^{*}, x_{i 2}^{*}, \ldots, x_{i N}^{*}$, where $x_{i 1}^{*}$ and $x_{i N}^{*}$ are the left- and right-end points of $A_{i}^{*}$, i.e., $a_{i 1}^{*}$ and $a_{i 4}^{*}$, respectively. The discretized points in the horizontal component, i.e., $x_{i j}^{*}$, are computed using Eq. (5). On the other hand, the discretized points in the vertical component, i.e., $\mu_{A_{i}^{*}}\left(x_{i j}^{*}\right)$, are computed using Eq. (1). The discretized points for $A_{i}^{*}$ are expressed in Eqs. (6) and (7) as follows. 


$$
\begin{gathered}
x_{i j}^{*}=x_{i 1}^{*}+(j-1)\left(\frac{x_{i N}^{*}-x_{i 1}^{*}}{N-1}\right), \\
x^{*}=\left[\begin{array}{ccc}
x_{11}^{*} & \cdots & x_{1 N}^{*} \\
\vdots & \ddots & \vdots \\
x_{m 1}^{*} & \cdots & x_{m N}^{*}
\end{array}\right], \\
\mu_{A_{i}^{*}}\left(x^{*}\right)=\left[\begin{array}{ccc}
\mu_{A_{i}^{*}}\left(x_{11}^{*}\right) & \cdots & \mu_{A_{i}^{*}}\left(x_{1 N}^{*}\right) \\
\vdots & \ddots & \vdots \\
\mu_{A_{i}^{*}}\left(x_{m 1}^{*}\right) & \cdots & \mu_{A_{i}^{*}}\left(x_{m N}^{*}\right)
\end{array}\right],
\end{gathered}
$$

where $i=1,2, \ldots, m, j=1,2,3, \ldots, N$.

Step 3: Compute the centroid of $A_{i}^{*}$ (i.e., $c X_{A_{i}^{*}}, c Y_{A_{i}^{*}}$ ). In this paper, the geometric centroid [15] is adopted, whereby $c X_{A_{i}^{*}}$ and $c Y_{A_{i}^{*}}$ are obtained with Eqs. (8) and (9), as follows.

$$
\begin{gathered}
c X_{A_{i}^{*}}=\frac{1}{6 L_{A_{i}^{*}}} \sum_{j=1}^{N}\left(x_{i j}^{*}+x_{i(j+1)}^{*}\right)\left(x_{i j}^{*} \mu_{A_{i}^{*}}\left(x_{i(j+1)}^{*}\right)+x_{i(j+1)}^{*} \mu_{A_{i}^{*}}\left(x_{i j}^{*}\right)\right), \\
c Y_{A_{i}^{*}}=\left\{\begin{array}{r}
\frac{1}{6 L_{A_{i}^{*}}^{*}} \sum_{j=1}^{N}\left[\left(\mu_{A_{i}^{*}}\left(x_{i j}^{*}\right)+\mu_{A_{i}^{*}}\left(x_{i(j+1)}^{*}\right)\right)\left(x_{i j}^{*} \mu_{A_{i}^{*}}\left(x_{i(j+1)}^{*}\right)-x_{i(j+1)}^{*} \mu_{A_{i}^{*}}\left(x_{i j}^{*}\right)\right)\right], \text { if } x_{i 1}^{*} \neq x_{i N}^{*} \\
\frac{\mu_{A_{i}^{*}}\left(x_{i}^{*}\right)}{2}, \text { if } x_{i 1}^{*}=x_{i N}^{*}
\end{array}\right.
\end{gathered}
$$

where $L_{A_{i}^{*}}=\frac{1}{2} \sum_{j=1}^{N}\left(x_{i j}^{*} \mu_{A_{i}^{*}}\left(x_{i(j+1)}^{*}\right)-x_{i(j+1)}^{*} \mu_{A_{i}^{*}}\left(x_{i j}^{*}\right)\right), i=1,2,3, \ldots, m, j=1,2,3, \ldots, N$.

Step 4: Refine the centroid (i.e., $c X_{A_{i}^{*}}, c Y_{A_{i}^{*}}$ ) using Eqs. (10)-(13) to obtain the new centroid, i.e., $c X_{A_{i}^{*}}^{*}, c Y_{A_{i}^{*}}^{*}$.

$$
\begin{gathered}
\bar{X}_{A_{i}^{*}}=\frac{1}{N} \sum_{j=1}^{N} x_{i j}^{*}, \\
\delta_{A_{i}^{*}}=\sqrt{\frac{1}{N-1} \sum_{j=1}^{N}\left(x_{i j}^{*}-\bar{X}_{A_{i}^{*}}\right)^{2}}, \\
c X_{A_{i}^{*}}^{*}=c X_{A_{i}^{*}}, \\
c Y_{A_{i}^{*}}^{*}=\frac{H_{i}}{2}-\delta_{A_{i}^{*}} c Y_{A_{i}^{*}},
\end{gathered}
$$

where $\delta_{A_{i}^{*}}$ denotes a dispersion coefficient of $A_{i}^{*}$, and $H_{i}=\max _{j=1,2, \ldots, N}\left\{\mu_{A_{i}^{*}}\left(x_{i j}^{*}\right)\right\}$, $i=1,2,3, \ldots, m$.

Step 5: Compute the ordering index, $I_{A_{i}^{*}}$, using Eqs. (14)-(15) as follows.

$$
\begin{gathered}
\varphi_{A_{i}^{*}}=\left\{\begin{array}{r}
1, \operatorname{sign}\left(c X_{A_{i}^{*}}^{*}\right) \geq 0 \\
-1, \operatorname{sign}\left(c X_{A_{i}^{*}}^{*}\right)<0
\end{array},\right. \\
I\left(A_{i}^{*}\right)=\varphi_{A_{i}} \sqrt{c X_{A_{i}^{*}}^{* 2}+c Y_{A_{i}^{*}}^{* 2}},
\end{gathered}
$$


where $\varphi_{A_{i}^{*}}$ indicates the position of the fuzzy numbers. $I\left(A_{i}^{*}\right)$ is the distance between point $\left(c X_{A_{i}^{*}}^{*}, c Y_{A_{i}^{*}}^{*}\right)$ and the origin $(0,0)$. A larger ordering index (i.e., $\left.I\left(A_{i}^{*}\right)\right)$ indicate a higher the ranking order.

\section{Experimental Study}

In this section, benchmark examples from [8] are used to evaluate the proposed method for ranking fuzzy numbers. An analysis of P1-P7 [5-9] is further reported.

\subsection{Ranking Generalized Fuzzy Numbers}

An example from [8], as depicted in Fig.1, is considered. Fuzzy membership functions for $A_{1}$ and $A_{2}$, are as follows.

$$
\begin{gathered}
\mu_{A_{1}}(x)=\left\{\begin{aligned}
x-1, & 1 \leq x \leq 2, \\
3-x, & \leq x \leq 3, \\
0, & \text { otherwise },
\end{aligned}\right. \\
\mu_{A_{2}}(x)=\left\{\begin{array}{r}
{\left[1-(x-2)^{2}\right]^{1 / 2}, 1 \leq x \leq 2,} \\
{\left[1-\frac{1}{4}(x-2)^{2}\right]^{1 / 2}, 2 \leq x \leq 4,} \\
0, \text { otherwise. }
\end{array}\right.
\end{gathered}
$$

The fuzzy numbers, $A_{1}$ and $A_{2}$ in Fig. 1, are transformed into normalized fuzzy numbers (i.e., $A_{1}^{*}, A_{2}^{*}$ respectively), as illustrated in Fig.3.

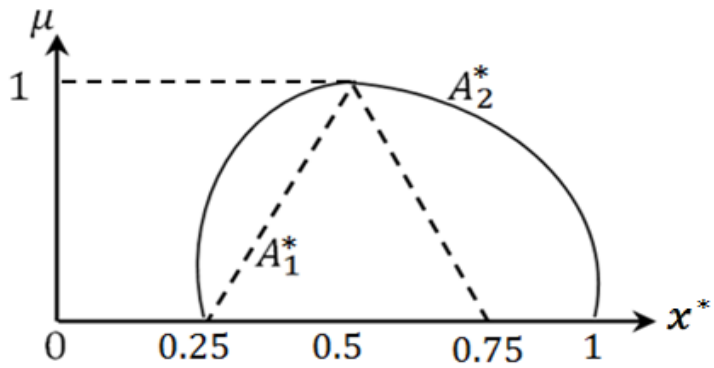

Fig. 3. Normalized triangular (i.e., $A_{1}^{*}$ ) and generalized (i.e., $A_{2}^{*}$ ) fuzzy numbers [8]

With the proposed method, $I\left(A_{1}^{*}\right)=0.669$ and $I\left(A_{2}^{*}\right)=0.723$ i.e., $I\left(A_{1}^{*}\right)<$ $I\left(A_{2}^{*}\right)$; therefore $A_{1}^{*} \preccurlyeq A_{2}^{*}$ and $A_{1} \preccurlyeq A_{2}$. The ranking results are in agreement with those in [8]. 


\subsection{Analysis of the Seven Reasonable Ordering Properties}

Three fuzzy numbers $A_{1}, A_{2}$, and $A_{3}$, are considered, where $A_{1}^{*}, A_{2}^{*}$, and $A_{3}^{*}$ are normalized fuzzy numbers. An ideal fuzzy ranking method should comply with the seven reasonable ordering properties [5-9] as follows:

P1: $\quad I\left(A_{1}^{*}\right) \geq I\left(A_{2}^{*}\right)$ occurs if $A_{1} \geqslant A_{2}$ and $I\left(A_{2}^{*}\right) \geq I\left(A_{1}^{*}\right)$ occurs if $A_{2} \geqslant A_{1}$. Therefore, $I\left(A_{1}^{*}\right)=I\left(A_{2}^{*}\right)$ is always true, if $A_{1} \sim A_{2}$.

P2: $\quad I\left(A_{1}^{*}\right) \geq I\left(A_{2}^{*}\right)$ occurs if $A_{1} \geqslant A_{2}$ and $I\left(A_{2}^{*}\right) \geq I\left(A_{3}^{*}\right)$ occurs if $A_{2} \geqslant A_{3}$. Therefore, $I\left(A_{1}^{*}\right) \geq I\left(A_{3}^{*}\right)$ is always true, for $A_{1} \geqslant A_{2} \geqslant A_{3}$.

P3: If $A_{1} \cap A_{2}=\emptyset$, and if $A_{1}$ is on the right side of $A_{2}$, then $I\left(A_{1}^{*}\right)>I\left(A_{2}^{*}\right)$ is always true.

P4: $\quad I\left(A_{1}^{*}\right)$ and $I\left(A_{2}^{*}\right)$ are computed separately to order $A_{1}$ and $A_{2}$. Therefore, the ranking outcome is not affected by other fuzzy numbers under comparison.

P5: If $A_{1} \geqslant A_{2}$, then $I\left(A_{1}^{*}\right) \geq I\left(A_{2}^{*}\right)$. An addition of $A_{3}$ to $A_{1}$ and $A_{2}$ changes the centroids of $A_{1}$ and $A_{2}$ correspondingly. fore, $I\left(A_{1}+A_{3}{ }^{*}\right) \geq I\left(A_{2}+A_{3}{ }^{*}\right)$, if $A_{1}+A_{3} \geqslant A_{2}+A_{3}$.

P6: If $A_{1} \geqslant A_{2}, I\left(A_{1}^{*}\right) \geq I\left(A_{2}^{*}\right)$. Similar to P5, a multiplication of $A_{3}$ to $A_{1}$ and $A_{2}$ changes the centroids of $A_{1}$ and $A_{2}$ correspondingly. Therefore, $I\left(A_{1} A_{3}{ }^{*}\right) \geq I\left(A_{2} A_{3}{ }^{*}\right)$, if $A_{1} A_{3} \geqslant A_{2} A_{3}$.

P7: If $A_{1} \geqslant A_{2} \geqslant A_{3}$, then $I\left(A_{1}^{*}\right) \geq I\left(A_{2}^{*}\right) \geq I\left(A_{3}^{*}\right)$. Consider $\varphi_{A_{1}^{*}}=\varphi_{A_{2}^{*}}=$ $\varphi_{A_{3}^{*}}=1$ for $A_{1}, A_{2}$ and $A_{3}$. For $-A_{1},-A_{2}$ and $-A_{3}, \varphi_{-A_{1}^{*}}=\varphi_{-A_{2}^{*}}=$ $\varphi_{-A_{3}^{*}}=-1$. Therefore, $-I\left(-A_{1}^{*}\right) \leq-I\left(-A_{2}^{*}\right) \leq-I\left(-A_{3}^{*}\right)$, for $-A_{1} \preccurlyeq$ $-A_{2} \preccurlyeq-A_{3}$.

The proof for P1-P4 is straightforward. Analysis of P5, P6 and P7 are more complicated, and are further illustrated with an example in [8]. The details are presented in the following section.

\subsection{An Empirical Study}

Consider an example in [8], $A_{1}=(2,4,4,6) ; A_{2}=(1,5,5,6)$, and $A_{3}=(3,5,5,6)$ as shown in Fig. 4(a). $A_{1}+A_{3}, A_{2}+A_{3}, A_{1} A_{3}$, and $A_{2} A_{3}$ (as depicted in Fig. 4(b) and Fig. 4(c)) are computed using fuzzy addition and fuzzy multiplication as summarized in Eqs. (3) and (4). Fuzzy images of $A_{1}, A_{2}$, and $A_{3}$ are presented in Fig. 4(d). Fuzzy numbers in Fig. 4 are normalized and presented in Fig. 5. 


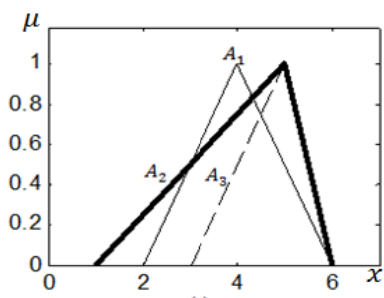

(a)

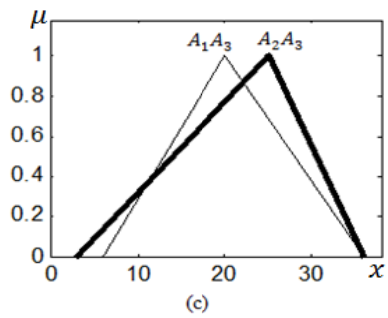

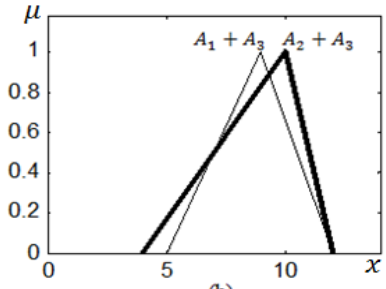

(b)

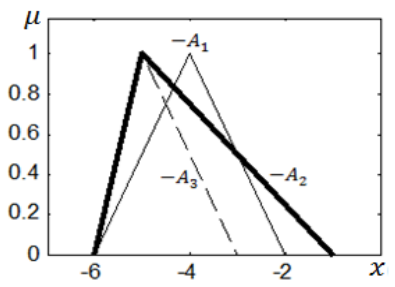

(d)

Fig. 4. (a) Three fuzzy numbers, $A_{1}, A_{2}$, and $A_{3}$, (b) fuzzy addition, $A_{1}+A_{3}$ and $A_{2}+A_{3}$, (c) fuzzy multiplication, $A_{1} A_{3}$ and $A_{2} A_{3}$, and (d) fuzzy images $-A_{1},-A_{2}$ and $-A_{3}$

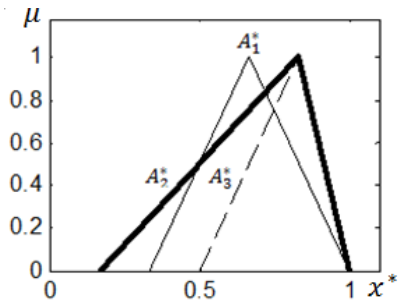

(a)

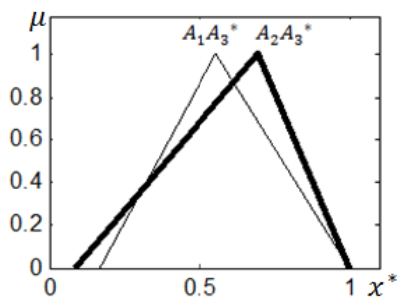

(c)

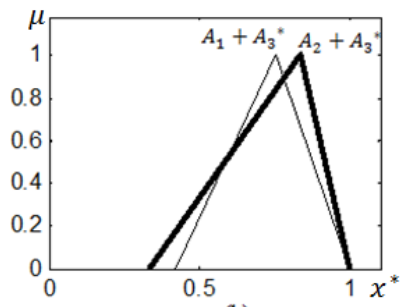

(b)

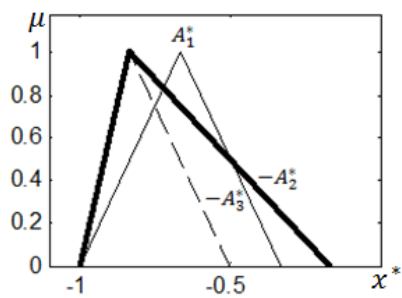

(d)

Fig. 5. Normalized fuzzy numbers of fuzzy numbers in Fig.4

The objective of this section is to analyze P5, P6, and P7 empirically. In this paper, $N=11$ is considered. The experimental results are summarized in Table 1. Columns " $I\left(A_{1}^{*}\right)$ ", " $I\left(A_{2}^{*}\right)$ ", and " $I\left(A_{3}^{*}\right)$ " show the ordering indexes for $A_{1}, A_{2}$, and $A_{3}$, respectively. Columns " $I\left(A_{1}+A_{3}{ }^{*}\right)$ " and " $I\left(A_{2}+A_{3}{ }^{*}\right) "$ show the ordering indexes for $A_{1}+A_{3}$ and $A_{2}+A_{3}$, respectively. Columns "I $\left(A_{1} A_{3}{ }^{*}\right)$ " and "I $\left(A_{2} A_{3}{ }^{*}\right)$ " show the ordering indexes for $A_{1} A_{3}$ and $A_{2} A_{3}$, respectively. Columns

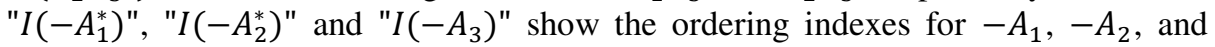
$-A_{3}$, respectively. 
Table 1. Ranking results of the proposed method

\begin{tabular}{|c|c|c|c|c|c|c|c|c|}
\hline$I\left(A_{1}^{*}\right)$ & $I\left(A_{2}^{*}\right)$ & $I\left(A_{3}^{*}\right)$ & $I\left(A_{1}+A_{3}^{*}\right)$ & $I\left(A_{2}+A_{3}^{*}\right)$ & $I\left(A_{1} A_{3}{ }^{*}\right)$ & $I\left(A_{2} A_{3}{ }^{*}\right)$ & $I\left(-A_{1}^{*}\right)$ & $I\left(-A_{3}^{*}\right)$ \\
\hline 0.791 & 0.782 & 0.872 & 0.827 & 0.822 & 0.687 & 0.684 & $-0.791-0.782$ & -0.872 \\
\hline \multicolumn{3}{|c|}{$A_{2} \preccurlyeq A_{1} \preccurlyeq A_{3}$} & \multicolumn{2}{|c|}{$A_{2}+A_{3} \preccurlyeq A_{1}+A_{3}$} & \multicolumn{2}{|c|}{$A_{2} A_{3} \preccurlyeq A_{1} A_{3}$} & \multicolumn{2}{|c|}{$-A_{3} \preccurlyeq-A_{1} \preccurlyeq-A_{2}$} \\
\hline
\end{tabular}

P5, P6 and P7 can be observed from Table 1. With the proposed method, $I\left(A_{1}^{*}\right)=0.791, I\left(A_{2}^{*}\right)=0.782, \quad I\left(A_{3}^{*}\right)=0.872 ;$ as such $A_{2} \preccurlyeq A_{1} \preccurlyeq A_{3} ; I\left(A_{1}+\right.$ $\left.A_{3}{ }^{*}\right)=0.827$, and $I\left(A_{2}+A_{3}{ }^{*}\right)=0.822$; therefore $I\left(A_{1}+A_{3}{ }^{*}\right)>I\left(A_{2}+A_{3}{ }^{*}\right)$ is satisfied. On the other hand, $I\left(A_{1} A_{3}{ }^{*}\right)=0.687$ and $I\left(A_{2} A_{3}{ }^{*}\right)=0.684$; therefore $I\left(A_{1} A_{3}{ }^{*}\right)>I\left(A_{2} A_{3}{ }^{*}\right)$ is satisfied. Lastly, $I\left(-A_{1}^{*}\right)=-0.791, I\left(-A_{2}^{*}\right)=-0.782$ and $I\left(-A_{3}^{*}\right)=-0.872$; therefore $-A_{3} \preccurlyeq-A_{1} \preccurlyeq-A_{2}$. In short, P5, P6 and P7 are satisfied.

\section{$5 \quad$ Concluding Remarks}

In this paper, a new fuzzy ranking method is proposed. It constitutes a solution for ranking fuzzy numbers. The proposed method has been empirically analyzed using benchmark examples. The seven reasonable ordering properties i.e., P1-P7 [5-9], have been satisfied empirically. The rationale and implications of the proposed method have also been analyzed and discussed.

For future work, the proposed method can be extended to measure similarity between fuzzy numbers. Application of the proposed method to decision making [17] will also be investigated.

\section{References}

1. Zhang, F., Ignatius, J., Lim, C.P., Zhao, Y.: A new method for ranking fuzzy num-bers and its application to group decision making. Appl. Math. Model. 38, 1563-1582 (2014)

2. Flaig, A., Barner, K.E., Arce, G.R.: Fuzzy ranking: theory and applications. Signal Proces. 80, 1017-1036 (2000)

3. Wang, Y.M., Chin, K.S., Poon, G.K.K., Yang, J.B.: Risk evaluation in failure mode and effects analysis using fuzzy weighted geometric mean. Expert Syst. Appl. 36, 1195-1207 (2009)

4. Lin, F.T.: Fuzzy job-shop scheduling based on ranking level ( ) interval-valued fuzzy numbers. IEEE Trans. Fuzzy Syst. 10, 510-522 (2002)

5. Wang, Z.X., Liu, Y.J., Fan, Z.P., Feng, B.: Ranking LR fuzzy number based on deviation degree. Inform. Sciences 179, 2070-2077 (2009)

6. Wang, Y.M., Luo, Y.: Area ranking of fuzzy numbers based on positive and negative ideal points. Comput. Math. Appl. 58, 1769-1779 (2009)

7. Asady, B.: The revised method of ranking LR fuzzy number based on deviation degree. Expert Syst. Appl. 37, 5056-5060 (2010)

8. Yu, V.F., Chi, H.T.X., Shen, C.: Ranking fuzzy numbers based on epsilon-deviation degree. Appl. Soft Comput. 13, 3621-3627 (2013) 
9. Abbasbandy, S., Asady, B.: Ranking of fuzzy numbers by sign distance. Inform. Sciences 176, 2405-2416 (2006)

10. Wang, Z.X., Liu, Y.J., Fan, Z.P., Feng, B.: Ranking LR fuzzy number based on deviation degree. Inform. Sciences 179, 2070-2077 (2009)

11. Chen, S.J., Chen, S.M.: Fuzzy risk analysis based on the ranking of generalized trapezoidal fuzzy numbers. Appl. Intell. 26, 1-11 (2007)

12. Dat, L.Q., Yu, V.F., Chou, S.Y.: An improved ranking method for fuzzy numbers based on the centroid-index. J. Intell. Fuzzy Syst. 14, 413-419 (2012)

13. Deng, Y., Zhenfu, Z., Qi, L.: Ranking fuzzy numbers with an area method using radius of gyration. Comput. Math. Appl. 51, 1127-1136 (2006)

14. Chai, K.C., Tay, K.M., Lim, C.P.: A new fuzzy ranking method using fuzzy prefer-ence relations. In: IEEE International Conference on Fuzzy Systems (FUZZ), pp. 1-5 (2014)

15. Bourke, P.: Calculating the area and centroid of a polygon (1988),

http: / /www.seas.upenn.edu/ sys502/extra_materials / Polygon\%20Area\%20and\%20Centroid.pdf

16. Abbasbandy, S., Hajjari, T.: A new approach for ranking of trapezoidal fuzzy numbers. Comput. Math. Appl. 57, 413-419 (2009)

17. Chen, S.M., Sanguansat, K.: Analyzing fuzzy risk based on a new fuzzy ranking method between generalized fuzzy numbers. Expert Syst. Appl. 38, 2163-2171 (2011)

18. Chai, K.C., Tay, K.M.: A perceptual computing-based approach for peer assessment. In: IEEE International Conference on System of Systems Engineering (SoSE), pp. 1-6 (2014) 